Document downloaded from:

http://hdl.handle.net/10251/77509

This paper must be cited as:

Bonet Aracil, MA.; Bou Belda, E.; Monllor Pérez, P.; Gisbert; Jaime (2016). Binder effectiveness of microcapsules applied onto cotton fabrics during laundry. The Journal of the Textile Institute. 107(3):300-306. doi:10.1080/00405000.2015.1029808.

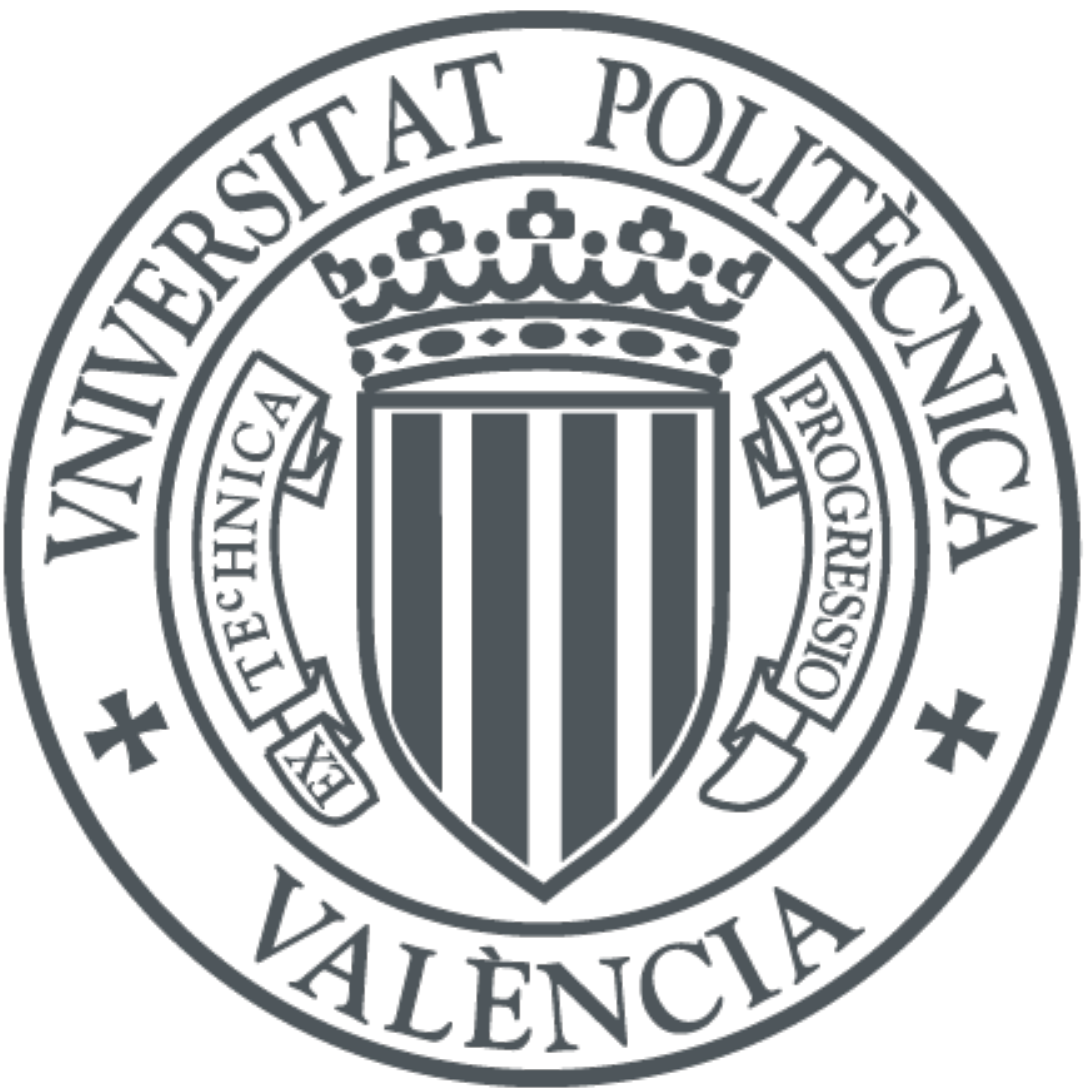

The final publication is available at

http://dx.doi.org/10.1080/00405000.2015.1029808

Copyright Taylor \& Francis

Additional Information 


\section{BINDER EFFECTIVENESS OF MICROCAPSULES APPLIED ONTO COTTON FABRICS DURING LAUNDRY}

Mª ÁNGELES BONET*, EVA BOU, PABLO MONLLOR, JAIME GISBERT,

M. Bonet ${ }^{1 \star}$, E. Bou-Belda ${ }^{1}$, P.Monllor ${ }^{1}$, J. Gisbert ${ }^{2}$,

${ }^{1}$ Universitat Politècnica de Valencia

Plaza Ferrandiz y Carbonell s/n

03801 Alcoy.Spain

${ }^{2}$ INNOVATEC SC,S.L.

Avenida de Elche $n^{0} 3$

03801 Alcoy

Spain

*To whom correspondence should be sent: maboar@txp.upv.es

M. Angeles Bonet Aracil

\section{AKNOWLEDGEMENTS}

Authors gratefully acknowledge the financial support received by this research project from the Spanish government in the programme "Plan Nacional 2008-2011" reference Mat 2009-14210-C02-01. 


\title{
BINDER EFFECTIVENESS OF MICROCAPSULES APPLIED ONTO COTTON FABRICS DURING LAUNDRY
}

\begin{abstract}
Microcapsules can be added to fabric in industrial processes however, they have not been widely spread among industrial companies. In this study we suggest the possibility of reloading microcapsules onto a fabric while cloths are washed. The effectiveness of different resins when microcapsules are applied in washing machine during domestic laundry process has been studied. Microcapsules containing lavender fragrance and melamine formaldehyde shell where adhered to the fabric by means of one acrylic acid as a resin, or some crosslinking agents such as butanetetracarboxylic acid (BTCA) or succinic acid (SUC). In order to evaluate their behaviour some laundering or ironing tests were conducted according to international standards (ISO). Every sample from the laboratory was studied with Scanning Electron Microscopy (SEM) and with a particle size counter. As a result we could observe which was the most suitable auxiliary used to bond microcapsules to fabric, and conclude about the conditions (temperature, concentration, etc.) in which we obtained the optimal results. It was demonstrated that domestic laundry is a suitable process to incorporate microcapsules to garments.
\end{abstract}

\section{KEYWORDS}

Cotton, Microcapsules, laundry, binder, SEM. 


\section{INTRODUCTION}

Microcapsules products were born with carbonless copying paper (Zhang, Saunders \& Thomas, 1999) and became considerably important in the pharmaceutical field (Stolnick, 1995). In other areas such as textiles they were firstly considered some time ago (Gordon, 2001). The main advantage of microcapsules is that new functions can be added and confer more properties such as cosmetic (Gisbert, Ibañez, Bonet, Monllor, Díaz, Montava, 2009), fragrances (Li, Lewis, Stewart, Qian, \& Boyter, 2006; Nelson, 2001; Monllor, Bonet, Cases, 2007; Monllor, Bonet, Sánchez, Cases, 2009; Monllor, Capablanca, Bonet, Gisbert, Díaz; Montava, 2010), phase change materials (Fan, Zhang, Wang, Li\& Zhu, 2004; Hawlader, Uddin, Khin, 2003; Xiao_Zheng, Zhi-Cheng, Guang-Long, Li-Xian \& Tao 2003; Zhang et al, 2004, Karthukeyan, Ramachandran, \& Shanmugasundaram, 2014) etc.

The most extended microcapsules applied to textiles are based on non reactive shells. In order to improve durability to washing or handle some auxiliary products are required to join microcapsules onto the fibres surface (Nelson, 2001); they are usually based on acrylic, polyurethanes, or silicone resins (Li et al. 2008; Rodrigues et al, 2009). Some studies show how polycarboxylic acids can be used in encapsulation process (Pascu, Garcia-Valls, \& Giamberini, 2008) or as a binder to join microcapsules (Voncina, Kreft, Kokol, Chen, 2009; Badulescu, Vivod, Jausovec, Voncina, 2008; Ziming, Genquan, Wai-man, Huiyi, 2011) or nanoparticles to fabrics (Montazer, Lessan, Moghadam, 2012; Wanga, Chen, 2005). 
To date the most industrial methods known to apply finishing treatments to fibres are padding, bath exhaust, coating or spraying. The main characteristic of bath exhaustion is that it is useful when chemical reaction can be established, and as we have remarked usually microcapsules' shell show no reactivity with fibres. Some authors demonstrated that bath exhaustion was not the most suitable procedure for that kind of products (Bonet, Capablanca, Monllor, Diaz, Montava, 2012). However, microcapsules are part of the recipe of some soaps (EP 0376385 A2) or in some cationic softeners (US 6,620,77 B2) what mean that those products can be applied to fabrics in laundry, which involves bath exhaustion procedure. Some of them show a recipe without binder and some of them include mainly acrylic resins (US 6,620,77 B2). Moreover, some patents show a thermal treatment in an automatic dryer at a temperature between 38$100^{\circ} \mathrm{C}$ after a treatment in a pre-soaking/washing process (USP 4,234,627).

It can be noticeable that there is no study about the binder effectiveness or the influence of ironing in crosslinking. The aim of this paper is to study the effectiveness of different resins when microcapsules are applied in washing machine while laundry. This work will study how many laundry cycles allow the microcapsules to remain on the fabric when they had been pasted onto the fibres while clothes were washed. Apart from laundry behaviour, change in colour will be evaluated in order to determine if the procedure has secondary effects such as yellowing.

\section{MATERIALS AND METHODS}




\subsection{Materials}

Microcapsules (CENTER FINISH 164/02 LAVANDA) were supplied by COLOR CENTER (Tarrasa, Spain). The wall material was melamine formaldehyde, and the microcapsules contained lavender fragrance. No further information was supplied by the provider. In order to bond the microcapsules to the fabric, an acrylic resin was applied, also supplied by Color Center and two polycarboxylic acids, butanetetracarboxylic acid (BTCA) from Alfa Aesar and Succinic acid (SUC) from Panreac. Polycarboxylic acids recquire the presence of a catalyst. It was sodium hypophosphite monohidrate (SHP) which was supplied from Alfa Aesar.

The fabric used was a $100 \%$ cotton twill fabric with $210 \mathrm{~g} / \mathrm{m}^{2}$, which had been chemically bleached with peroxide in an industrial process.

\subsection{Adhering microcapsules onto fabrics.}

The equipment used to apply microcapsules was a domestic washing machine. Its capacity was $5 \mathrm{Kg}$ and its maximum volume of water was $11 \mathrm{~L}$.

Microcapsules dispersion was prepared in a glass vessel. Four tests were conducted. One test was comprised of no binder. The rest contained BTCA, SUC, or acrylic resin. The supplier suggested concentrations to use. Different samples were prepared depending on the binder nature. All the samples contained $5 \%$ owf (over weight of fibre) of microcapsules. One of the baths was comprised of microcapsules without any binder. The recipes for samples with 
polycarboxylic acids (BTCA and SUC) as binder were formulated with $5 \%$ owf of microcapsules $4 \%$ owf of binder and $2 \%$ owf of catalyst. The test with acrylic binder (RES) was based on $5 \%$ owf of microcapsules and $12,5 \%$ owf of binder. As soon as the washing machine finished, the fabrics were dried in an IR dryer at $100^{\circ} \mathrm{C}$ until the fabric was completely dry. Lately, fabrics should be thermally treated so as to polymerize the binder. Commercial brands suggest different temperatures but in this study the curing temperature has been selected in order to get the minimum yellowness on the cotton fibre and different curing temperatures have been evaluated.

Table 1 summarises the treatment conditions herein studied.

\section{Insert table 1 about here}

\subsection{Scanning Electron Microscopy (SEM).}

For surface observation, a scanning electron microscope (SEM) Phenom microscope (FEl Company) was used. Each sample was fixed on a standard sample holder and sputter coated with a gold -platinum mixture. Samples were then examined with suitable accelerating voltage and magnification.

\subsection{Microcapsules permanence.}

To test the effectiveness of the binders some laundering tests were conducted. The specimens were treated on a short time program in a Heraeus Linitest for 
45 min at $30{ }^{\circ} \mathrm{C}$, in accordance with ISO Standard $105 \mathrm{C} 01$. Just finishing one cycle, samples were dried on a horizontal surface at room temperature. All samples were examined after 1, 2, 3, 4 and 5 cycles.

\subsection{Counting microcapsules.}

The particle size distribution of the microcapsules was measured by a Coulter ${ }^{\circledR}$ Counter apparatus (Multisizer Z2, Coulter Electronics, Northwell, UK). The particle size was expressed as the equivalent volume diameter and two replicates were performed for each batch of microcapsule, to reduce error an average curve was calculated and analysed.

This method was used to measure the microcapsules dispersion just immediately it was prepared and the wastewater from the washing machine. The number of microcapsules remaining on the fabric was calculated as the difference between the number of microcapsules on the prepared bath and the number of microcapsules in wastewater.

Furthermore, the wastewater from ISO laundry was also measured to observe the microcapsules behaviour in each laundry cycle.

2.6.- Ironing samples. 
Some samples were ironed at different temperatures (110, 150 and $\left.200^{\circ} \mathrm{C}\right)$. The ironing procedure was performed as UNE EN ISO 105 X11 standard indicated.

\section{RESULTS}

\section{1.- Curing temperature.}

Polycarboxylic acids should be cured at temperatures from $150^{\circ} \mathrm{C}$ in order to induce crosslinking. Knowing that thermal treatment on cellulosic fibres is responsible of yellowness, different curing temperatures have been evaluated. Figure 1 shows the whiteness index depending on the binder used and the temperature of treatment. It could be predictable tough, the white colour on cotton fibres decreases as the temperature increases, and it can be appreciated that when temperature is higher than $160^{\circ} \mathrm{C}$ cotton fibre whiteness considerably decreases. When fabrics are treated with binders the whiteness index, at lower temperatures than $140^{\circ} \mathrm{C}$, is approximately the same than the fabric without treatment. As a result $150^{\circ} \mathrm{C}$ has been considered a good temperature for curing polycarboxylic acids.

It is considerably noticeable that when temperature is lower that $160^{\circ} \mathrm{C}$, samples treated with acrylic binder show the lowest values of whiteness index. However, at about $160^{\circ} \mathrm{C}$ the whiteness index is approximately the same for every fabric which had been treated with a binder, and slightly lower than the index for cotton without treatment. 


\section{Insert figure 1 about here}

\section{2.- Binder effectiveness}

Microcapsules dispersion was added on washing machine, lately fabrics were examined by SEM and it could be observed that all the samples showed some microcapsules on its surface, including the ones from test one (M25) where no binder had been used. Images show not sensible differences between samples obtained from different binders. Thus, only one fabric is shown in Figure 2 where it is shown the sample BTCA- 150 .

Insert figure 2 about here

In order to observe the binder effect we analysed images from washed samples as standard ISO 105 C10:2007 indicates. When we compare figure 2 with figure 3 the laundering effect can be clearly seen, some microcapsules have been removed form the fabric surface. If the binder effect is analysed (see figure 3a and $3 b$ ), it can be easily appreciated that binder plays its roll avoiding microcapsules to scape from the fabric's surface. This result is not surprising however, it is not only important the binder presence but the curing temperature for polycarboxylic acids too. The influence of curing temperature has been demonstrated as fabrics cured at $150^{\circ} \mathrm{C}$ show a wider number of microcapsules on its surface than the ones without any curing, it is obvious when comparing figure $3 \mathrm{c}$ (BTCA without temperature) with $3 d$ (BTCA at 150을 C) or $3 e$ (SUC without temperature) with $3 f\left(S U C\right.$ at $150^{\circ}$ C). Moreover, 
considering the sample cured at $150^{\circ} \mathrm{C}$, see figure $3 \mathrm{e}$ and $3 \mathrm{f}$, samples treated with SUC are the ones that show the higher quantity of microcapsules after having washed fabrics during 5 cycles.

\section{Insert figure 3 about here}

Despite the fact that results from SEM can be obvious, the analysis from wastewater can clarify those results as it is more objective and shows the results expressed in the quantity of particles that wastewater contained.

In order to study the laundry effect and binder efficiency, microcapsules deposition on fabric should be characterised. Consequently, the number of microcapsules on fabric surface was calculated. Results were obtained by the difference in the number of particles in water previously to add the fabric and the number of particles in wastewater. Particles, which were not in wastewater, can only have been placed onto the fabrics. Knowing the area of the treated fabric, we could estimate the number of microcapsules (MIC) in a square meter $\left(\mathrm{m}^{2}\right)$. Moreover, the number of particles in wastewater after each laundry cycle was obtained. Figure 4 shows the behaviour of the microcapsules while washing the treated fabrics. It evidences the influence of the binder in microcapsules permanence on fabric previously to have it washed. 
It can be clearly observed a decreasing tendency in the number of microcapsules that remain on the fabric when the number of cycles increases. That behaviour evidences the necessity of adding any kind of binder if it is desired to increase the microcapsules presence on fabrics. Samples with no binder $(25 \mathrm{M})$ do not show any microcapsule between their fibres. This result is in accordance with the ones showed by SEM technique in figure 3. Furthermore, if resin has been added to act as a binder, thermal treatment should be performed otherwise microcapsules would be lost in the first or second cycle (see figure 4 SUC and SUC-150). Samples treated with succinic acid (SUC) have lost all the microcapsules in the second cycle, whereas when the sample binder (SUC-150) has been treated at $150^{\circ} \mathrm{C}$ a large number of microcapsules still remain on fabric's surface after 5 washing cycles. However, when acrylic binder is added and thermal treatment is applied, it loses more quantity of microcapsules than succinic acid after the third cycle. This means that succinic acid shows the best results regarded the permanence of microcapsules on fabrics when it is properly cured. Samples treated with BTCA and washed could not be evaluated as some microcapsules stick together in the bath in presence of BTCA. Consequently, it is not possible to measure them. This test has been performed twice and the same behaviour was observed. Moreover, it is remarkable that when microcapsules dispersion was prepared with acrylic binder (RES) and non-distilled water was used some aggregates could be observed. The aggregates presence can be due to some kind of ionic behaviour what makes difficult to have it evaluated. If microcapsules have the property of getting stuck together when used with acrylic resin or BTCA binder, there is no possibility in obtaining an accurate procedure, as it is not possible to 
repeat it with the same result. This means that succinic acid is the most suitable for applying microcapsules on laundry in processes where current water is used.

\section{2.- Fabric yellowing}

In order to determine the influence of the treatment on the fabrics the whiteness index was measured. Table 2 shows either the white index values for each sample and the difference in white between the treated samples compared with the cotton fabric without being treated.

\section{Insert table 2 about here}

It is interesting to note that microcapsules on fabric (25M) change the whiteness of the original fabric. However, if we compare all the measurements slight differences between the different used binders can be appreciated. When measurements are referred to differences $(\Delta \mathrm{WI} \mathrm{CIE})$ it can be observed than both BTCA-150 and SUC-150 are the ones with higher values. However, in order to evaluate if measured values would be observed by human eye, colorimetric values have been analysed and the difference between each sample and the untreated cotton have been calculated. One of the colour systems on which most chromatic studies are based is the CIE $L^{*} a^{*} b^{*}$ system (Aspland, 1993; Billmeyer, Saltzman, 1982). When we observe chromatic values, we can appreciate that $L^{*}$ value (darkness/lightness) can be considered 
constant around 95. Thus, implies no changes because of the treatment have been induced in fabric lightness. When differences are calculated a clear correlation was found between $\Delta \mathrm{WI} \mathrm{CIE}$ and $\Delta \mathrm{Ea}^{*} \mathrm{~b}^{*}$, as it could be predicted. The single most striking observation to emerge from the data comparison is that samples with polycarboxylic acids (BTCA-150 and SUC-150), which include microcapsules, show higher witnesses index than cotton treated at the same temperature without microcapsules and no polycarboxylic acids treatments (cotton 150). Colour differences from samples with microcapsules shown in Table 2 demonstrate that despite the fact that spectrophotometer measurements show objective differences, it can be stated that they cannot be appreciated by human eye as they are not higher than 1 .

\section{3.- Ironing as thermal treatment for crosslinking.}

In order to determine if thermal treatment while ironing process would be able to induce crosslinking, samples after being treated in washing machine were ironed at different temperatures value as the Standard establishes. Latter on, they were washed as UNE EN ISO 105 suggests so as to check if crosslinking by ironing is effective or not.

\section{Insert figure 5 about here}

It can be clearly observed that after 1 laundry cycle, samples still show microcapsules however it is noticeable that the ones treated at $110^{\circ} \mathrm{C}$ have lost the majority of the microcapsules if compared with figure 2. By contrast, when 
samples ironed at temperatures of $150^{\circ} \mathrm{C}$ and $200^{\circ} \mathrm{C}$ are studied results are really encouraging as a wide number of microcapsules still remain on fabric surface.

\section{CONCLUSIONS}

This study was set out to determine if washing machine is a suitable device to apply microcapsules onto fabrics despite the use of any kind of binder agent.

First of all, the present study compares acrylic resin with two polycarboxylic acids and the thermal treatment to cure the binder agent. It is not surprising that microcapsules remain on fabric surface when the treatment includes some binder as it improves microcapsules adhesion onto the fibre. It is noticeable that thermal treatment is required if the microcapsule effect should last more than one washing cycle. Otherwise, nor binder nor thermal treatment should be applied to the fabric.

As a consequence of thermal treatment, colour changes can be induced on cotton fibre. Chromatic studies have been conducted in order to determine either the thermal influence or the presence of chemicals can produce on a noticeable change in colour on fabrics. The evidence from this study suggests that some changes occur, as it was predictable. However, either differences in witnesses index and chromatic differences demonstrate they are not sensitive enough to be observed by human eye. 
When acrylic resin is compared with polycarboxylic acids and considering thermal conditions for each one, the evidence shows that the later allow microcapsules to remain on the fabric the longer. Apparently, succinic acid displays the higher number of microcapsules on the fibre. Furthermore, acrylic binder develops some aggregates of microcapsules and makes impossible to test the binder appropriately.

To sum up, when applying microcapsules to fabrics by washing machine, succinic acid if cured at $150^{\circ} \mathrm{C}$ shows the best results and treated fabric has not changed sensitively the colour. Ironing at $150^{\circ} \mathrm{C}$ is effective in the curing process and in the appropriate binding of microcapsules in fabrics. This article demonstrates the possibility of applying microcapsules to fabrics in a domestic process using succinic acid in a conventional washing procedure and ironing with domestic equipment.

\section{AKNOWLEDGEMENTS}

On title page in order to avid providing authors information. 


\section{REFERENCES}

Aspland JR, (1993) Color, Color Measurement and Control. Textile chemists and colorists, 25(1):34-41

Badulescu, R.; Vivod, V.; Jausovec, D.; \& Voncina, B.; Grafting of ethyllcellulose microcapsules onto cotton fibers. Carbohydrate Polymers, 71.2008.85-91

Billmeyer WF, Saltzman S. (1982) Principles of Color Technology, $2^{\text {nd }}$ edition. John Wiley\&Sons, New York

Bonet, M.; Capablanca, P.; Monllor, P.; Diaz, P.; Montava, I. 2012. Studing bath exhaustion as a method to apply microcapsules on fabrics. Journal of the textile institute.;103,6:629-935.

Fan, Y.F., Zhang, X.X., Wang, X.C. Li, J., \& Zhu, Q.B. 2004. Super-cooling prevention of microencapsulated phase change material. Thermochimica Acta $4131-6$.

Hawlader, M.N.A., Uddin, M.S. and Khin,M.M. 2003. Microencapsulated PCM thermal-energy storage system. Applied Energy 74. 195-202.

Karthukeyan, M.; Ramachandran, T. \& Shanmugasundaram, O.L. 2014, Synthesis, characterization, and development of thermally enhanced cotton 
fabric using nanoencapsulated phase change materials containing paraffin wax. Journal of the Textile Institute 105.12.1279-1286.

Gisbert, J., Ibañez F., Bonet, M., Monllor, P., Díaz, P. \& I. Montava.2009. Increasing hydration of the epidermis by microcapsules in sterilized products. Journal of applied polymer science.113.4. 2282-2286

Li, S., Lewis, J.E., Stewart, N.M., Qian, L. \& Boyter, H. 2008. Effect of finishing methods on washing durability of microencapsulated aroma finishing. Journal of the Textile Institute 99.2.177-183.

Monllor, P., Bonet, \& M., Cases, F. 2007. Characterization of the behaviour of flavour microcapsules in cotton fabrics. European Polymer Journal.43.24812490.

Monllor, P. Bonet, M.; Sanchez, L. \& Cases, F. (2009). Thermal behaviour of microencapsulated flavours when applied to cellulosic fabrics. Textile research journal, 79.4. 365-380

Monllor, P., Capablanca, L., Bonet, M., Gisbert, J., Díaz, P., \& Montava, I. 2010.Improvement of microcapsules adhesion to fabrics. Textile research journal.80.7. 631-635

Montazer, M., Lessan, F., Moghadam, M.B. 2012. Nano-TiO2/maleic acid/triethanol amine/sodium hypophosphite colloid on cotton to produce cross- 
linking and self-cleaning properties. The journal of the textile institute; 103(8):795-805.

Nelson G., 2001. Microencapsulates in textile finishing Rev. Prog. Color. 31.5764.

Pascu, O; Garcia-Valls, R.; \& Giamberini, M.. (2008)Interfacial polymerization of an epoxy resin and carboxylic acids for the synthesis of microcapsules. Polymer International 57. 995-1006

Rodrigues, S.N.; Martinis, I.M.; Fernandes, I.P.; Gomes, P.B.; Mata, V.G.; Barreiro, M.F,; \& Rodrigues, A.E. 2009. Scentfashion: Microencpasulated perfumes for textile application. Chemical engineering journal. 149.. 463-472.

Stolnik, S. 1995.Long circulating microparticulate drug carriers. Adv. Drug Delivery Rev. 16.195-214.

Voncina, B.; Kreft, O.; Kokol, V.; \& Chen, W.T. 2009. "Encapsulation of Rosemary Oil in ethylcellulose microcapasules. Textile and Polymer Journal vol1 No.1.

Wanga, C.C., Chen, C.C. 2005. Physical properties of the crosslinked cellulose catalyzed with nanotitanium dioxide under UV irradiation and electronic field. Applied Catalysis A: General; 293:171-179. 
Xiao-Zheng, L. Zhi-Cheng, T., Guang-Long, Z., Li-Xian, S \& Tao, Z. 2004. Microencapsulation of $n$-Eicosane as Energy Storage Material. Chinese Journal of Chemistry, 22, 411- 414 .

Zhang, Z.,Saunders, R., \& Thomas, C.R. 1999.Mechanical strength of single microcapsules determined by a novel micromanipulation technique. Journal of Microencapsulation 16 (1). 117-124.

Ziming, Y.; Genquan, L.; Wai-man, A.; \& Huiyi, Z. 2011. Advanced Materials Research. 221.308-315. 


\section{FIGURE CAPTIONS}

Figure 1.- Whiteness index depending on curing temperature

Figure 2.- Microcapsules applied on fabrics by washing machine process with BTCA binder, cured at $150^{\circ} \mathrm{C}$ and without any laundry.

Figure 3.- Fabrics treated by ISO 105 C10 after 5 washing cycles. a) 25M; b) RES; c) BTCA; d) BTCA-150; d) SUC; e) SUC-150.

Figure 4.- Microcapsules presence on the fabrics $\left(\mathrm{mics} / \mathrm{m}^{2}\right)$ after different laundry cycles.

Figure 5.- Fabrics treated by ironing and washed once. 


\section{FIGURES}

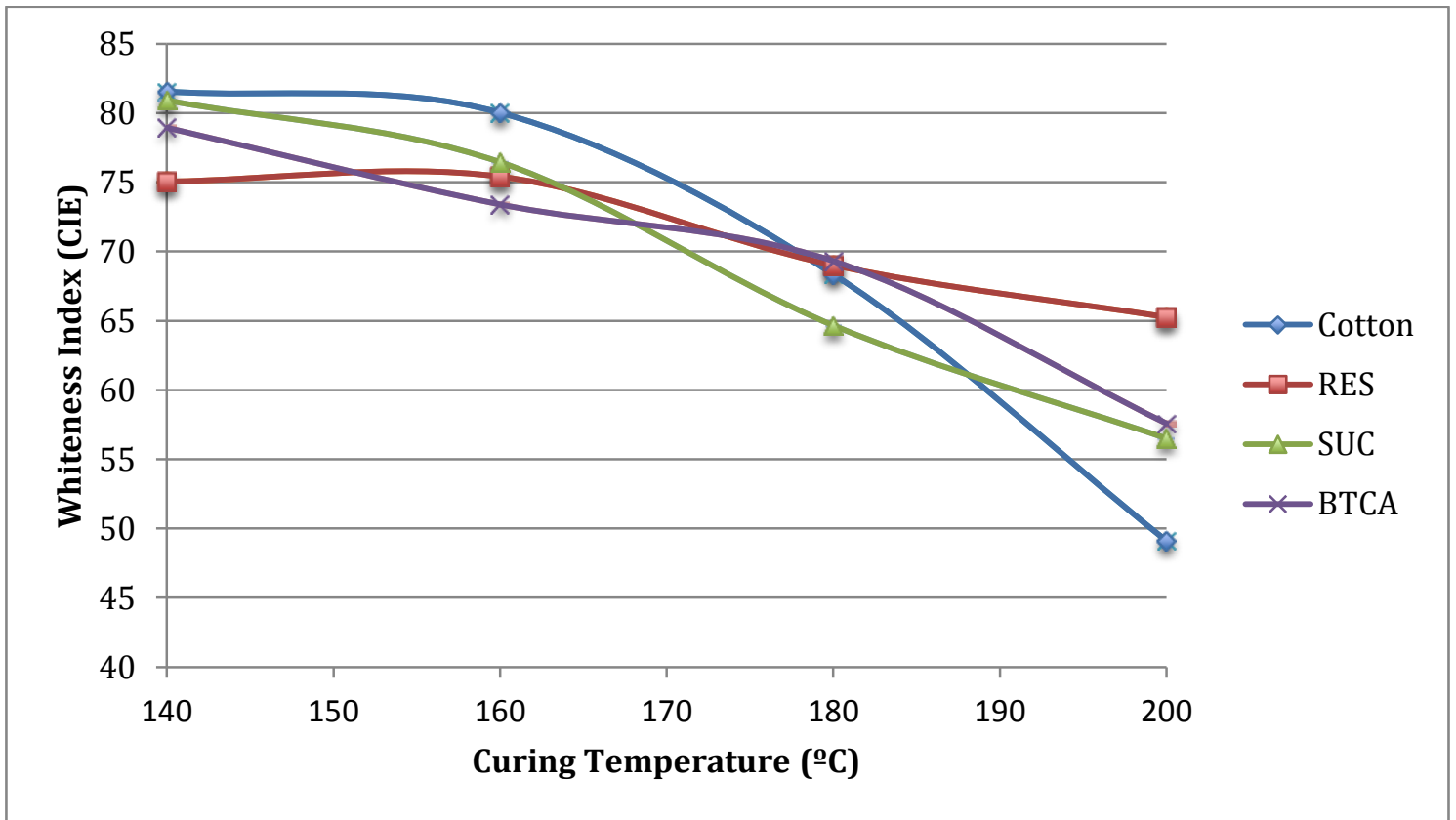

Figure 1.- Whiteness index depending on curing temperature 


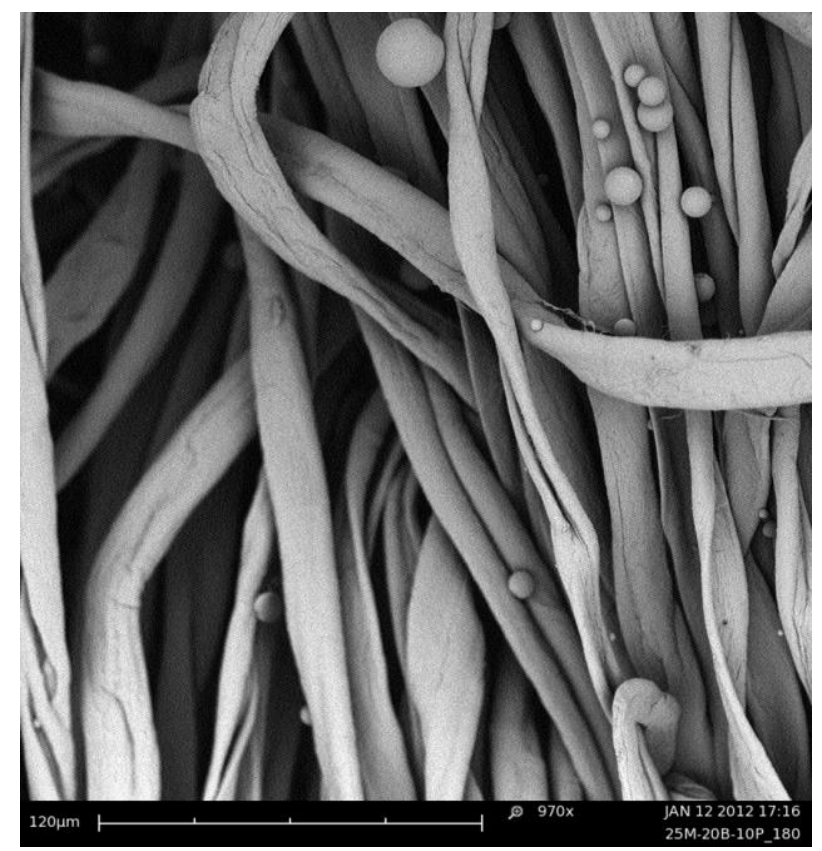

Figure 2.- Microcapsules applied on fabrics by washing machine process with BTCA binder, cured at $150^{\circ} \mathrm{C}$ and without any laundry. 

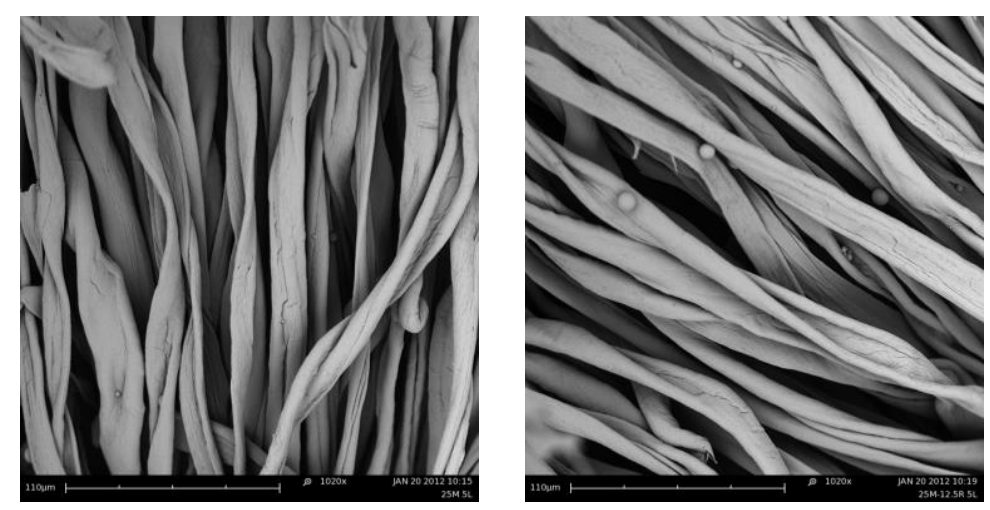
a) $25 \mathrm{M}$
b) RES
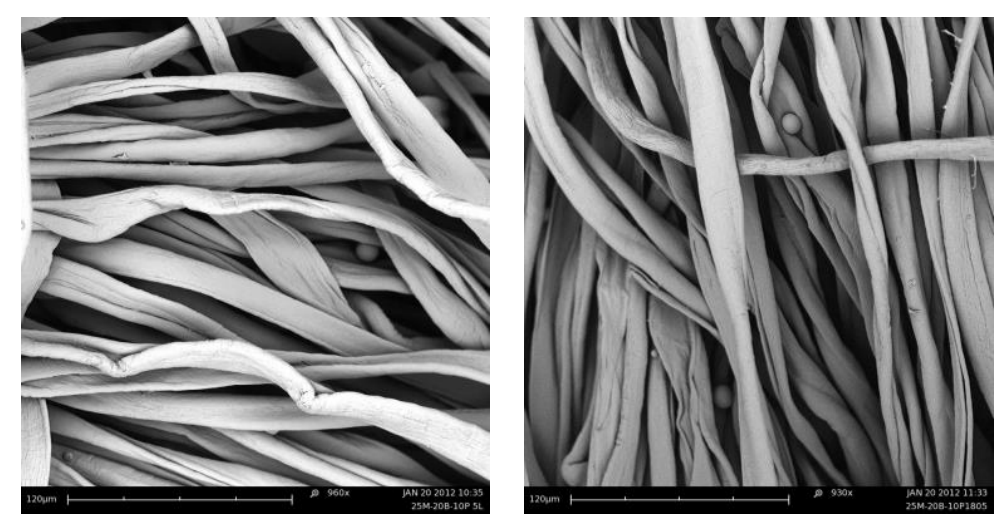
c) BTCA

d) BTCA-150
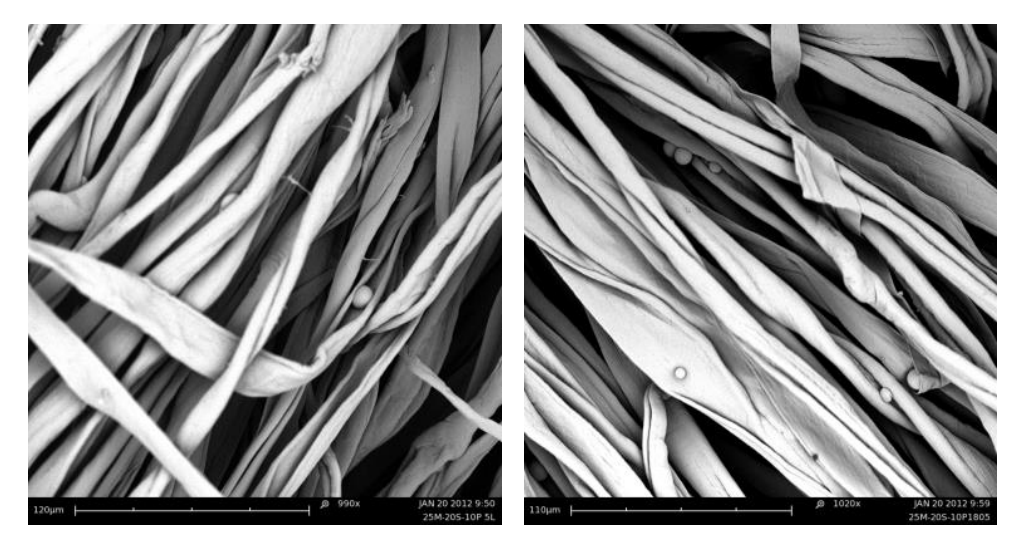

e) SUC

f) SUC-150

Figure 3.- Fabrics treated by ISO 105 C10 after 5 cycles. a) 25M; b) RES; c) BTCA; d) BTCA150; e) SUC; f) SUC-150. 


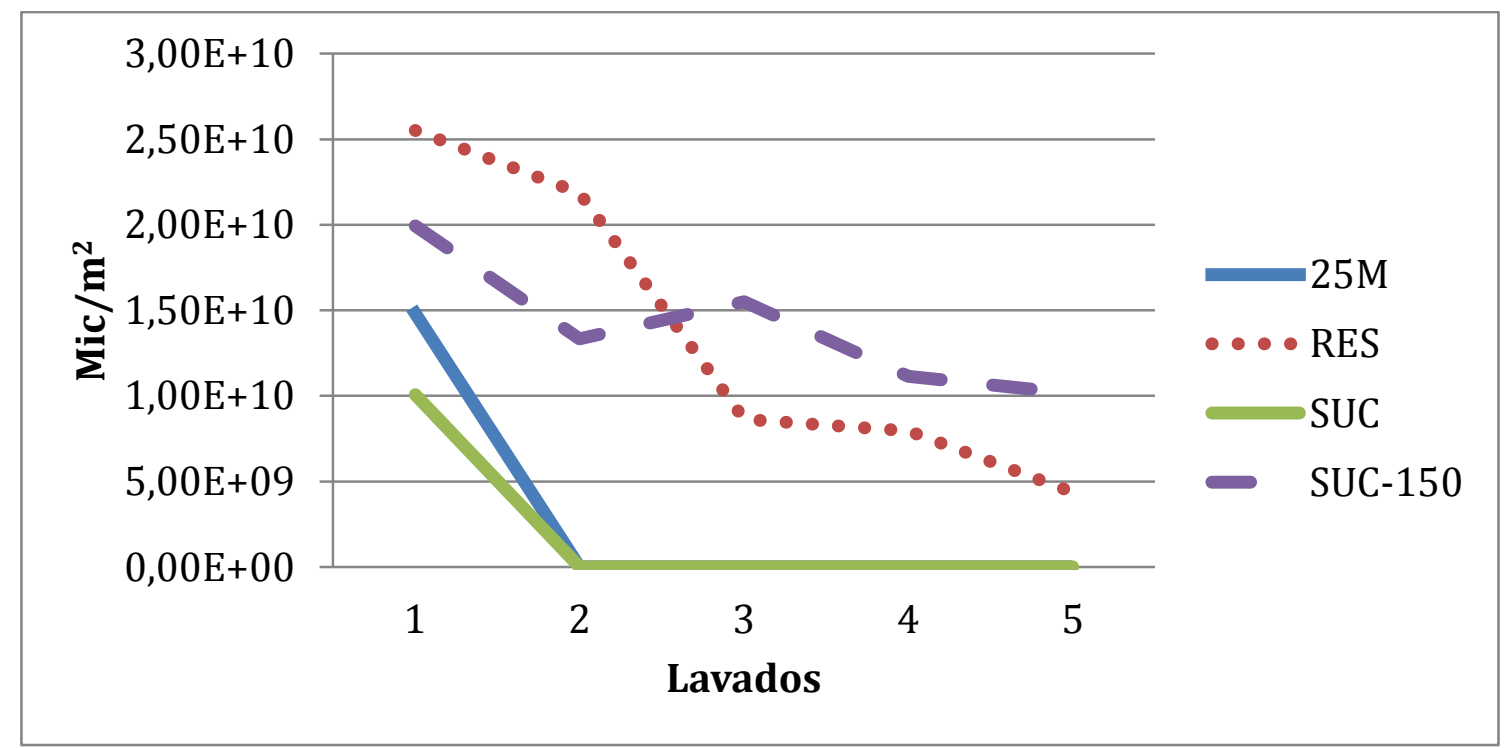

Figure 4.- Microcapsules presence on the fabrics $\left(\mathrm{mics} / \mathrm{m}^{2}\right)$ after different laundry cycles. 


\section{FIGURE 5}

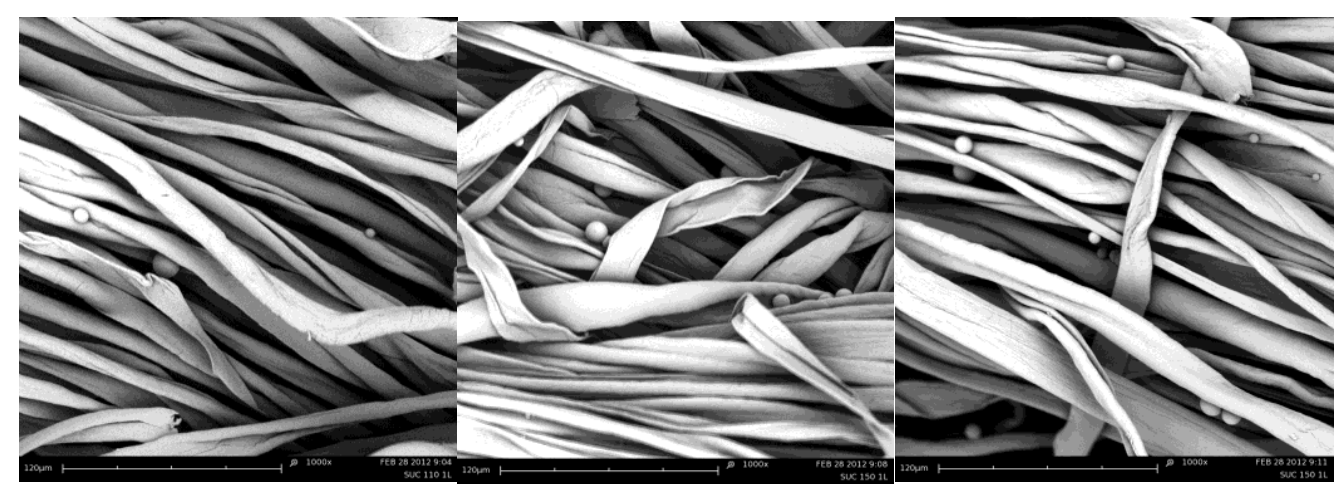
a) $110^{\circ} \mathrm{C}$
b) $150^{\circ} \mathrm{C}$
c) $200^{\circ} \mathrm{C}$

Figure 5.- Fabrics treated by ironing and washed once. a) ironed at $110^{\circ} \mathrm{C}$; b) ironed at $150^{\circ} \mathrm{C}$; c) ironed at $200^{\circ} \mathrm{C}$. 


\section{TABLES}

Table 1.- Laundry conditions

$\begin{array}{llllll}\text { TEST } 1 & \text { TEST } 2 & \text { TEST } 3 & \text { TEST } 4 & \text { TEST } 5 & \text { TEST } 6\end{array}$

Microcapsules

$\begin{array}{llllll}5 \% & 5 \% & 5 \% & 5 \% & 5 \% & 5 \%\end{array}$

concentration (o.w.f)

Binder

$0 \% \quad 20 \% \quad 20 \% \quad 20 \% \quad 20 \% \quad 2,5 \%$

concetration (o.w.f)

Binder composition

-- $\quad$ BTCA BTCA SUC SUC RES

Catalyst (owf)

$--\quad 2 \% \quad 2 \%$

Bath Temperature (응

30

30

30

$30 \quad 30$

30

Drying temperature ( $~ C$

110

110

110

110

110

110

Curing temperature $(\stackrel{\circ}{\circ})$

150

$150 \quad 110$

Curing time (seconds)

$--$

Referece

$25 \mathrm{M}$

$--$

$\begin{array}{llll}120 & 120 & 120 & 120\end{array}$

BTCA BTCA- SUC- SUC RES

$150 \quad 150$


Table 2.- White index values.

\begin{tabular}{lll}
\hline SAMPLE & WI CIE & $\Delta$ WI CIE \\
\hline Cotton & 80.99 & -- \\
$25 M$ & 78,96 & -2.03 \\
BTCA & 79.98 & -1.01 \\
BTCA-150 & 78.33 & -2.66 \\
SUC & 79.09 & -1.3 \\
SUC-150 & 76.63 & -4.36 \\
RES & 77.01 & -3.98 \\
\hline
\end{tabular}

